\title{
Review of: "Investigating surgical smoke in otolaryngology operating rooms"
}

\author{
Mark Ereth ${ }^{1}$ \\ 1 Mayo Foundation for Medical Education and Research
}

Potential competing interests: The author(s) declared that no potential competing interests exist.

The authors are to be commended for measuring PM 0.3 and 0.5--the most critical sizes--which impart risk to all OR personnel. Further, the use of local exhaust suction in the field adjacent to the cautery device is also important for protecting personnel. Of interest is the relatively high formaldehyde levels at baseline. These works emphasize the importance of surgical field suction exhaust and the use of other decontamination technologies that clear the breathing zone air of the surgery, anesthesia, and nursing teams. 